\title{
Chest Tube Malposition in Diaphragmatic Injury
}

\author{
Kang Kook Choi, Min A Lee, Gil Jae Lee, Byung Chul Yu, \\ Dae Sung Ma, Yang Bin Jeon, Jung Nam Lee \\ Department of Trauma Surgery, Gachon University Gil Medical Center, Incheon, Korea
}

In blunt trauma, diaphragmatic injuries are often overlooked. The repair of a diaphragmatic injury is not technically demanding in the acute phase. However, delayed diagnosis can cause serious complications such as respiratory compromise and incarceration of abdominal organs. We report a left diaphragmatic injury accompanied by herniation of the stomach.

(Trauma Image Proced 2016(1):14-15)

Key Words: Chest Tubes; Diaphragm; Injury; Thoracostomy; Hernia

\section{CASE}

A 67-year-old man was transferred to the emergency department by paramedics. The patient was involved in a traffic accident while driving and was entrapped in the crushed car when the paramedics arrived at the scene of the accident. The patient's mental status was stupor on arrival to the trauma center. His initial blood pressure could not be determined. His heart rate was $86 / \mathrm{min}$, respiratory rate was $32 / \mathrm{min}$, and body temperature was $35.4^{\circ} \mathrm{C}$. Endotracheal intubation was performed immediately. During resuscitation, bilateral tube thoracostomy was performed for bilateral chest wall crepitus. After the tube thoracostomy, chest radiography was performed (Fig. 1.). Chest computed tomography (CT) performed after stabilization of blood pressure (Fig. 2. and 3.) showed bilateral hemopneumothorax. The left chest tube was placed in the abdominal cavity (Fig. 4.).

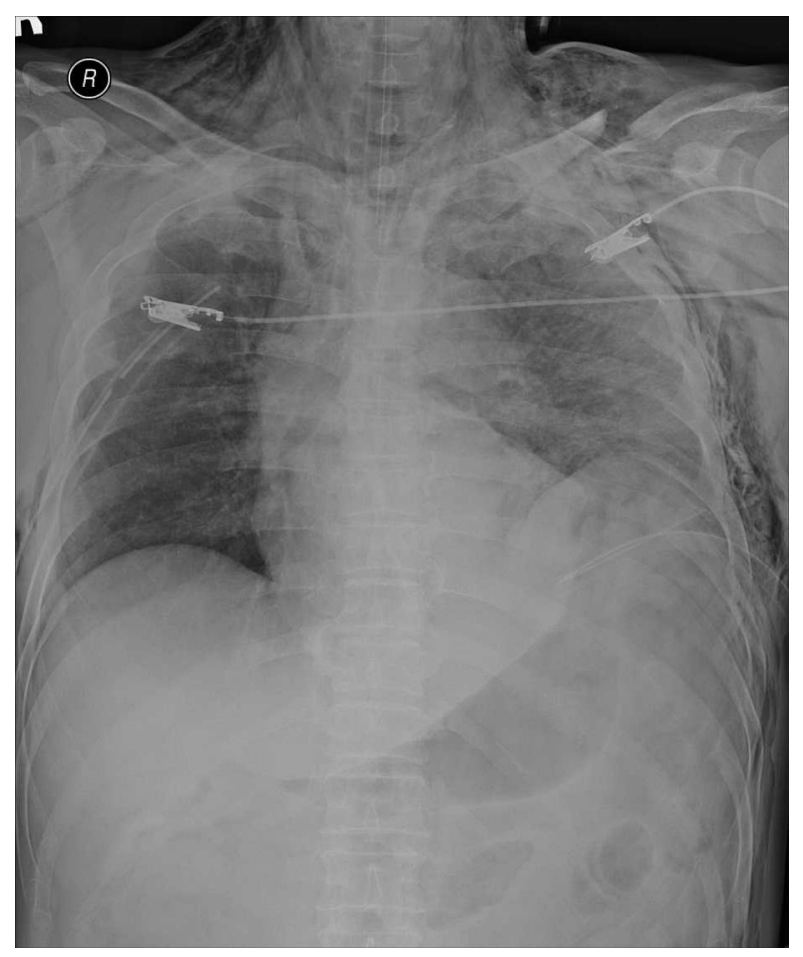

Fig. 1. Chest anteroposterior view radiograph shows elevated left diaphragm and unfavorable chest tube position.

Received: July 7, 2016 Revised: August 14, 2016 Accepted: August 14, 2016

Correspondence to: Jung Nam Lee, Department of Trauma Surgery, Gachon University Gil Medical Center, 21, Namdon-daero, 774 Beon-gil, Namdong-gu, Incheon 21565, South Korea

Tel: 82-32-460-3010, Fax: 82-32-460-2372, E-mail: jnlee@gilhospital.com

Copyright (c) 2016 Korean Association for Research, Procedures and Education on Trauma. All rights reserved.

(c) This is an open-access article distributed under the terms of the Creative Commons Attribution Non-Commercial License (http://creativecommons.org/ licenses/by-nc/4.0) which permits unrestricted noncommercial use, distribution, and reproduction in any medium, provided the original work is properly cited 


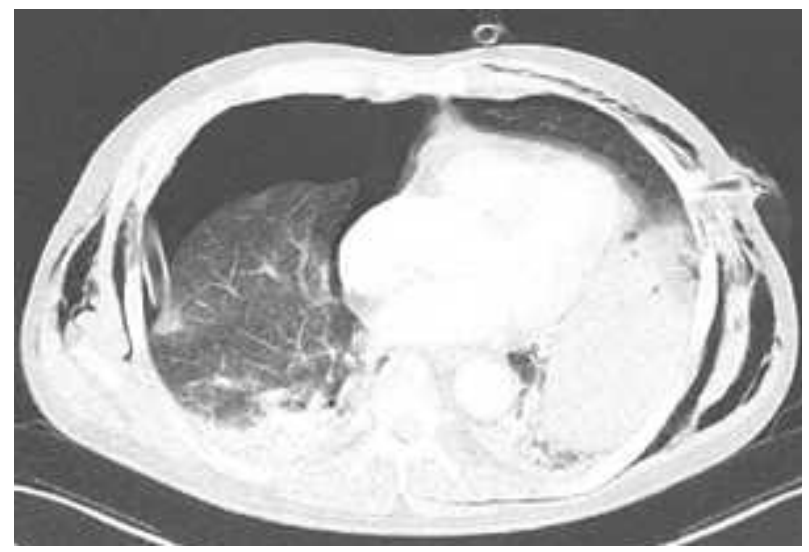

Fig. 2. Chest computed tomography shows bilateral hemopneumothorax.

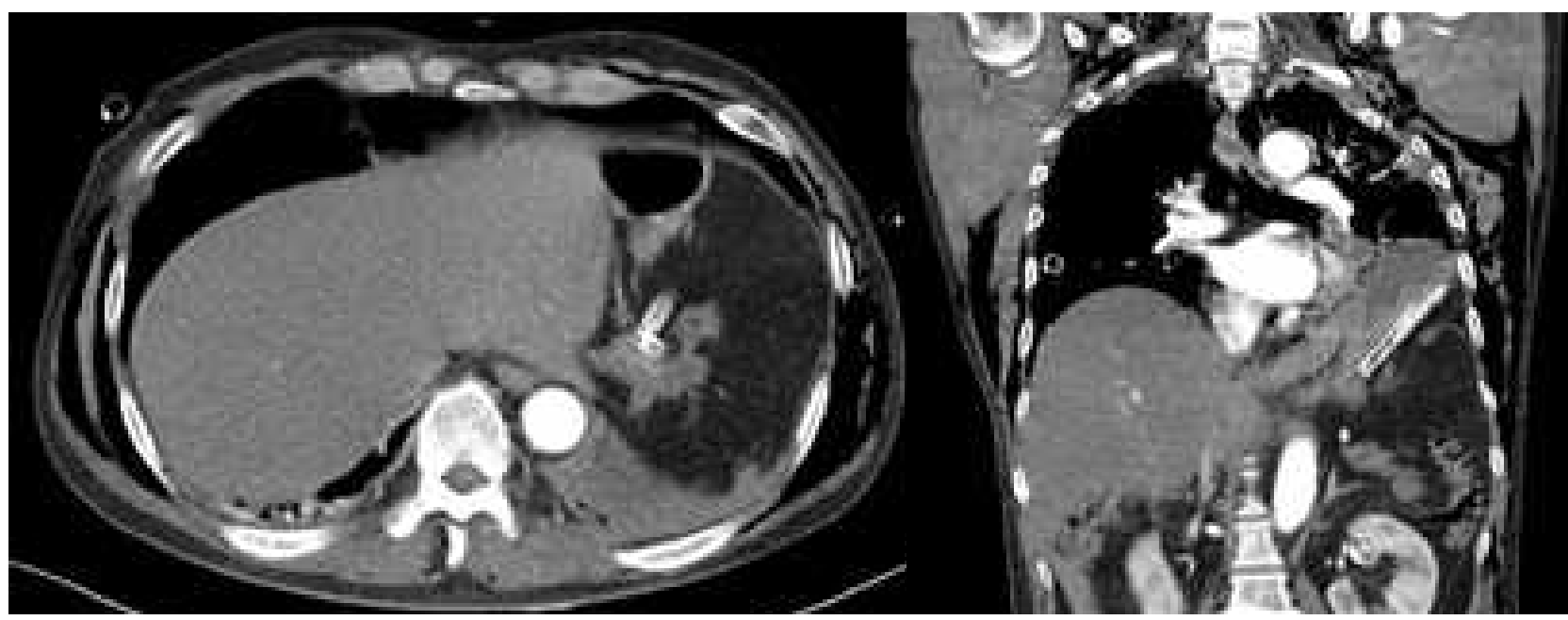

Fig. 3. Chest computed tomography shows left chest tube malposition.

\section{DISCUSSION}

Chest tube malposition can occur in diaphragmatic injury. Clinical suspicion is important for the diagnosis of diaphragmatic injury $(1,2)$.

\section{CONFLICT OF INTEREST}

No potential conflict of interest relevant to this article was reported.

\section{REFERENCES}

1. Bocchini G, Guida F, Sica G, Codella U, Scaglione M. Diaphragmatic injuries after blunt trauma: are they still a challenge? Reviewing CT findings and integrated imaging. Emergency radiology. 2012;19(3):225-35.

2. Hanna WC, Ferri LE. Acute traumatic diaphragmatic injury. Thoracic surgery clinics. 2009;19(4):485-9. 